\title{
A qualitative assessment of the role of a viscosity depending on the third invariant of the rate-of-deformation tensor upon turbulent non-Newtonian flow
}

\author{
P.J. Oliveira ${ }^{\text {a }}$, F.T. Pinho ${ }^{\text {b,* }}$ \\ a Departamento de Engenharia Electromecânica, Universidade da Beira Interior, Rua Marquês D'Ávila e Bolama, \\ 6200 Covilhã, Portugal \\ b Departamento de Engenharia Mecânica e Gestão Industrial, Faculdade de Engenharia, Rua dos Bragas, \\ 4099 Porto Codex, Portugal
}

Received 7 December 1996; received in revised form 16 November 1997

\begin{abstract}
The numerical simulation of some non-Newtonian effects in wall and wall-free turbulent flows, such as drag reduction in pipe flows or the decrease in transverse normal Reynolds stresses, has been attempted in the past with a limited degree of success on the basis of modified wall functions applied to traditional turbulence models $(k-\varepsilon)$, rather than through more realistic rheological constitutive equations. In this work, it is qualitatively shown that if the viscosity function of a generalised Newtonian fluid is assumed to depend on the third invariant of the rate of deformation tensor, there is an increase of the viscous diffusion terms, but especially, of the dissipation of turbulence kinetic energy by a factor equal to the Trouton ratio of the fluid, divided by the Trouton ratio of the solvent, thus indicating a possible way to improve rheological-turbulence modelling. (C) 1998 Elsevier Science B.V. All rights reserved.
\end{abstract}

Keywords: Turbulence; Strain-thickening viscosity

\section{Introduction}

In non-Newtonian fluid dynamics, there have been many observations of a reduction of the Reynolds stresses and the intensification of their anisotropy in both wall-free and especially in wall-dominated turbulent flows. Examples of the former are the works of Berman and Tan [1] on axisymmetric jets, and more recently, of Castro and Pinho [2] in an axisymmetric sudden expansion, whereas the classical example of the latter case is the well known drag reduction in

* Corresponding author. Fax: + 35122059125.

0377-0257/98/\$19.00 (C) 1998 Elsevier Science B.V. All rights reserved.

PII S0377-0257(97)00107-9 
turbulent pipe flow first reported by Toms in 1949. This reduction of the friction coefficient occurs in many non-Newtonian turbulent pipe flows and can reach maximum values of the order of $80 \%$. Its potential benefit to practical applications, such as district heating and cooling systems and other instances of transport of non-Newtonian fluids and energy in long pipes, fostered intensive research in the field from the late fifties onwards. The first important reviews on the subject were those of Hoyt [3] and Virk [4] in the seventies, when the first tentative theories to explain drag reduction were formulated.

Of the various theories that were developed, some did not sustain the proof of time. One of the earliest, still regarded as a serious possibility, although with changes and adaptations, was initially formulated by Lumley [5]. The proposed mechanism for drag reduction was an increased viscosity near the wall, caused by elongational deformation of the molecules by the turbulence. As the molecules are stretched, their resistance to extension increases, thus the higher viscosity. In laminar steady shear flows, stretching and rotation of molecules occur simultaneously, therefore, the molecules do not stay sufficient time under the stretching field and are not strongly elongated; however, under turbulent flow conditions, there are many instances of stretching without rotation and the molecules can be dramatically elongated.

The theory gained support as experimental results gathered information on the normal rates of deformation encountered by the polymer solutions flowing close to walls, as in the boundarylayer studies of the bursting processes by Reischman and Tiederman [6] and Luchik and Tiederman [7], amongst others. According to these authors, the resistance of the fluid molecules to normal deformations increases enormously and non-linearly with the strain rates; this ever increasing resistance to normal deformation interferes with the turbulence production and especially with the turbulence dissipation mechanisms. The measurements of Bewersdorff and Berman [8] showed a Reynolds stress deficit which the authors also related to an increased local viscosity in the buffer layer. Their theoretical predictions, assuming a pseudo-laminar velocity profile brought about by the high viscosity, were in agreement with the Reynolds stress deficit measurements. The work emphasised the need to correlate turbulence with the behaviour of polymer molecules, in particular the turbulent fluctuating strain rate and the molecular extension in the wall region. The direct proportionality between molecular extension and its resistance to further deformation is nowadays a well-known fact from molecular theory, (Larson [9]).

There are other reports in which drag reduction in turbulent flow is attributed to an increased local effective viscosity in the buffer layer: either associated with the alignment of fibres and rod-shaped particles in the wall region of turbulent pipe flows of fibres (Lumley [5]) and particles (Radin et al. [10]), or due to the increased air concentration near the wall because of aeration in open channel flows in spillways, or even due to the presence of suspended sediments (Chanson [11]).

The discovery of heterogeneous drag reduction, as reported by Bewersdorff [12], relaunched in the eighties, the discussion of drag reduction theories. Arguments based on the kinetics of the molecules were introduced in the development of drag reduction theories, leading to the quasi-static yo-yo model of Ryskin [13] and the affine deformation theory of Tabor-De Gennes (De Gennes [14] and Tabor et al. [15]). From all these theoretical models and observations, there are two main conclusions to be drawn: 
1. Elasticity definitely plays a role in drag reduction, but it is not clear at the end what is the parameter, the material function or the combination of those that best describes it. Elongational viscosity seems to be important, although, by itself, it is most probably insufficient to describe all observed flow features;

2. A comprehensive theory for explaining drag reduction and the behaviour of polymer solutions in wall-free flows requires the investigation of the interaction between the molecular dynamics and the flow field, and so it is intimately related to the development of more powerful and realistic rheological models for dilute solutions. The combination of rheology with turbulence models is therefore a powerful tool for understanding non-Newtonian turbulent flow hydrodynamics.

The adequate design of equipment and systems in process engineering requires the ability to predict the flow of non-Newtonian fluids in complex geometries. Now that surfactants are also known to exhibit drag and heat transfer reduction, with the added advantages of an almost permanent resistance to mechanical degradation and controllability of some of their non-Newtonian features (Kawaguchi et al. [16]), making them especially useful for large energy transport systems such as district heating and cooling (Steiff and Klöpper [17]), the interest on computational methods for the prediction of non-Newtonian fluid dynamics has intensified.

The first serious attempts to model numerically the turbulent pipe flow of drag reducing fluids were in the late seventies by Durst and Rastogi [18], Hassid and Poreh [19] and Durst et al. [20], who based their methods on various modifications of the standard $k-\varepsilon$ turbulence model. In these simulations, the non-Newtonian fluids were always very dilute aqueous polymer solutions of constant viscometric viscosity and the other non-viscous fluid flow characteristics, which contributed to drag reduction, were introduced via modified wall functions, rather than through the fluid constitutive rheological model. A decade later, Politis [21] and Collins [22] adapted the $k-\varepsilon$ model to variable viscometric viscosity fluids, relating its shear-thinning variation with the rate of dissipation of turbulent kinetic energy. However, as in the past, the non-viscous fluid features responsible for drag reduction continued to be implemented on the basis of modified wall functions, rather than on the basis of the fluid rheology.

The viscosity model adopted in those studies was the generalised Newtonian fluid model (GNF), but only with provision for a dependence of viscosity on the second invariant of the rate of deformation tensor. According to the definition of Reiner-Rivlin fluids, from which the GNF model can be derived (Astarita and Marrucci [23]), the material function viscosity of GNF fluids also depends on the third invariant, which is zero only if the flow is one or two-dimensional. It so happens that a turbulent flow is always three-dimensional, unless time-average quantities are referred, and consequently the third invariant does not vanish, contrary to the assumption of the former authors.

From the above, it is clear that for a proper rheological constitutive equation to handle turbulent flows, fairly more complicated models than the GNF are required, such as a complex multi-mode rheological model. Multi-mode models are complex and require an enormous computational power not yet easily available. Even for laminar flows of highly viscous fluids, where such constitutive equations are in use, their application is not straightforward and in many cases is still at an exploratory stage. In theory, such models would then be capable of simultaneously and accurately predicting the viscometric and elongational viscosities, among other relevant elastic material functions. Assuming that we would know the correct rheological 
constitutive equation, the task would still be enormous because it would involve developing a coupled turbulence-rheological model.

At the current stage of development and knowledge, this approach is also far too complex, because the adequate rheological models are unknown. A simpler alternative is the assumption of the relevance of a particular type of effect, which we know to be important from the existing theories of drag reduction, and the ensuing adoption of the simplest constitutive equation that can partly or on the whole account for that effect. Then, one would be able to gain insight into the physics of the phenomena, and expertise on the development of coupled turbulence-rheological models.

An obvious candidate for this methodology is a constitutive equation that essentially predicts correctly the viscometric and elongational viscosities, but such a model is not simple because it is already a differential equation on the stress. However, since there is very little experience on coupled rheology-turbulence modelling and the technological question of how to measure the correct elongational viscosity has not yet been definitely answered by the rheologists (Petrie [24]), the added complexity would not pay off at this stage. A simpler idea, adopted in this work and justified below, is to consider a generalized Newtonian model, with a viscosity depending on both the second and the third invariant of the rate of deformation tensor.

If indeed the normal rates of strain play an important role in turbulence, an equation that considers this dependence can in principle give some insight into this class of phenomena and enable predictions in the right direction. A qualitative assessment of the role of the viscosity function of the GNF model, with provision for a strain-thickening variation through the third invariant of the rate of deformation tensor, can give some ideas of its role upon turbulent flow. Of course, a more detailed and complete answer will need full results of numerical simulations, which is beyond the scope of this paper.

The importance of this simple approach has already been recognised by Den Toonder et al. $[25,26]$ and Orlandi [27], who used direct numerical simulations (DNS) to gain insight into the same phenomena. Their constitutive equations were also inspired by the GNF model with different viscosity functions and both predicted an increase of the viscosity with the strain rate. Orlandi [27] defined an anisotropic viscosity function with the influence of the polymer acting only in the direction of the normal stresses, which required the calculation of the ratio between the mean square rotation rate and the mean square strain rate. Initially, Den Toonder et al. [25] followed an approach similar to that adopted here, a single scalar viscosity function related to the second and third invariants of the rate of deformation tensor, but in the second paper [26] they combined it with the ideas of Orlandi regarding anisotropic behaviour, and investigated also an anisotropic viscoelastic model.

Due to limitations of the DNS procedures and the available computing power, Orlandi [27] and Den Toonder et al. [25,26] fell short of representing real flow conditions at high Reynolds numbers and only small drag reductions were attained. These works confirmed that the elongation rate plays an important role through the viscosity material function, with compression of molecules being at least as important as their stretching. High elongational viscosities, especially if acting in specific directions, were found to be necessary for drag reduction and were more important than memory effects. The constitutive equations adopted were rather simple and unable to explain some known rheological features of the fluids, but they clearly identified some effects and showed the need for more research using this approach before proceeding to the next level of complexity in rheological modelling applied to turbulent flows. 
The use of DNS for flow predictions is still limited to flows in very simple geometries at very low Reynolds numbers, and even then an enormous computing power is required. Real applications of CFD rely on less powerful computers, involve a higher degree of geometrical and fluid complexity and have to be performed in a considerably shorter period of time, thus resorting to single-point turbulence modelling. The combination of rheological and turbulence modelling is thus an essential tool for solving this problem, and can be considered a new area of research.

It is the objective of this paper to demonstrate qualitatively the usefulness of this coupled turbulence-rheology modelling approach when applied to turbulent non-Newtonian flows. To this aim, an order of magnitude analysis is applied to the various terms in the momentum and the modelled turbulence kinetic energy equations of a fluid which has a simple strain-thickening dependent viscosity and it is shown that a new term leads to a rate of dissipation much larger than the case of the fluid with a viscosity independent of the strain rate. A confirmation of effects in the right direction will make it worth further work on the development of turbulence models for this constitutive equation, with obvious extensions in the future to better and more complex rheological models.

In the next section, a discussion of the way to account for strain-thickening effects on the viscosity is undertaken. Then, the problem is formulated in the sense that the equations and terms to be investigated are presented. This will be followed by the order of magnitude analysis of those terms and the discussion of the results. The paper ends with a summary of the main conclusions. The development of the coupled rheology-turbulence model is postponed for a second report.

\section{The fluids}

This section presents and discusses information from the literature on the elongational viscosity of real dilute solutions and ends with the adoption of a simple constitutive equation.

\subsection{The elongational viscosity of drag reducing fluids}

To the authors knowledge, there are no reliable measurements of the elongational viscosity of fluids exhibiting drag reduction, just evidence that it may be an important parameter (Durst et al. [28] amongst others) and this stems from various difficulties:

1. Although the definition of elongational viscosity is well known, its practical implementation is not straightforward because of the need to attain a steady state during the measurement (Petrie [24]). Various technical approaches have been developed for the measurement of this material function and the results compared in order to assess the merits and disadvantages of each approach so that a standard procedure could be selected (Hudson and Jones [29] and the projects S1 and M1). A final answer to this problem is coming out slowly, but it is clear that in the short term, the outcome will not be a method as accurate as that used for the measurement of the viscometric viscosity, as the paper of Dontula et al. [30] on the shortcomings of the opposed-jet technique shows. 
2. For the very mobile systems that constitute the universe of the drag reducing fluids, the stresses involved in any of the existing methods are still too low to be accurately measured.

Recently, Hermansky and Boger [31] showed that the opposed jet technique was a promising method for implementation in commercial equipment for very mobile systems. However, the first measurements of fluids manufactured with drag reducing additives were those of Tirtaatmadja and Sridhar [32], who used a filament stretching technique to investigate dilute solutions of high molecular weight polyisobutylene in various viscous Newtonian solvents (Boger fluids). With this technique, the measured quantity approaches well the elongational viscosity, but a steady state condition is only attained for a limited range of strain rates. The transient Trouton ratio was measured and shown to increase from values of around three, proper of a Newtonian fluid, to more than one thousand. This value was considerably higher than those found with more concentrated polymer solutions because the molecules have more freedom to extend.

The opposed jet technique is more adequate for measuring a steady state quantity, but the deformation is non-homogeneous, the fluid is subject to a significant pre-shearing and is only approximately a pure elongation. Nevertheless, $\mathrm{Ng}$ et al. [33] used it to test various very dilute solutions in viscous Newtonian solvents $(50 \mathrm{ppm}$ by weight of $\mathrm{CMC}$, xantham gum, polyacrylamide and polyisobutylene). The additives investigated in this work are more typical of those encountered in drag reduction investigations although they were dissolved into high viscosity solvents. The fluids exhibited a constant viscometric viscosity (Boger fluids), but different behaviours as far as the steady state elongational viscosity is concerned, depending on the type of polymer structure, as shown in Fig. 1 which summarizes qualitatively those results.

Fig. 1 illustrates the behaviour of very dilute polymer solutions, which have a constant viscometric viscosity well above that of water. There is no reason a priori not to accept that similar behaviours will be found when the same additives are dissolved in water. These aqueous solutions, at concentrations up to about a few hundred ppm, also yield constant shear viscosity fluids, as can be confirmed in the works of Hoyt [3] and Virk [4], but at higher concentrations (from under $1000 \mathrm{ppm}$ ) the viscometric viscosity shows a shear-thinning pattern. Polymers such as polyethylene oxide or polyacrylamide are very efficient drag reducers, and aqueous solutions of these, at concentrations of $100 \mathrm{ppm}$, already exhibit drag reductions of the order of $70-80 \%$ (Berman [34]), values which are close to the maximum drag reduction asymptote of Virk et al. [35].

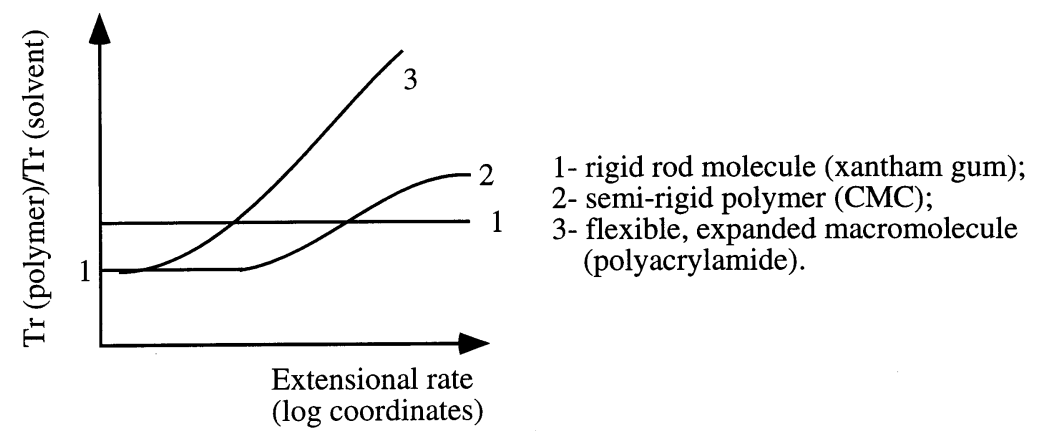

Fig. 1. Qualitative variation with the elongation rate of the Trouton ratio of polymer solutions relative to their solvents (from measurements of $\mathrm{Ng}$ et al. [33] for very dilute polymer solutions). 
In this work, it is desirable to separate shear and strain rate effects by assuming a shear-rate independent variation of the viscosity. As far as the steady state elongational viscosity is concerned, Fig. 1 identifies two main limiting cases: an elongational viscosity which is independent of the value of the strain rate (curve 1), and the elongational viscosity of very flexible polymers, which increases as a power law of the strain rate when this quantity exceeds a specific onset value (curve 3). The behaviour of curve 2 is intermediate to the previous two cases. Actually, curve 1 is idealized, because there must be a continuous variation of the elongational viscosity with the strain rate, starting from the Newtonian value of three times the shear viscosity in the limit of very low strain rates. In the paper of $\mathrm{Ng}$ et al. [33], such transition simply occurred at strain rate values below the lowest reported.

\subsection{The constitutive equation}

The simplest non-Newtonian fluid model that is able to accurately describe viscous effects is the GNF in which the stress tensor $(\boldsymbol{\sigma})$ is directly proportional to the rate of deformation tensor $\left(\boldsymbol{S} \equiv\left(\nabla \boldsymbol{u}+\nabla \boldsymbol{u}^{\mathrm{T}}\right) / 2\right)$ :

$$
\sigma=2 \mu S,
$$

where the viscosity function $\mu$ is in general a function of the three principal invariants of the rate of deformation tensor:

$$
\mu=\mu\left(I_{S}, I I_{S}, I I I_{S}\right) .
$$

Non-Newtonian liquids behave as incompressible fluids, thus $I_{S}=\operatorname{tr} S=0$, and in the laminar two-dimensional case the third invariant also vanishes $\left(I I I_{S} \equiv \operatorname{det} \boldsymbol{S}=0\right)$ which leaves the viscosity dependent only on the second invariant $\mu=\mu(\dot{\gamma})$ with $\dot{\gamma}=\sqrt{-4 I I_{S}}=\sqrt{2 S_{i j} S_{i j}}$.

Turbulent flows, however, are always locally three-dimensional, even when the flow is two-dimensional on the average, and if the normal rates of deformation play an important role in the mechanisms of turbulence generation, then the third invariant of the rate of deformation tensor should certainly be taken into consideration. For this reason, we keep an explicit dependence of viscosity on both the second and the third invariants of $\boldsymbol{S}$, which we write, instead, as

$$
\mu=\mu(\dot{\gamma}, \dot{\varepsilon}),
$$

where the shear parameter $\dot{\gamma}$ was defined above and the elongational parameter $\dot{\varepsilon}$ is given by

$$
\dot{\varepsilon}=\frac{6 \operatorname{det} S}{\operatorname{tr} S^{2}} .
$$

These parameters have a direct physical interpretation: in single shear flow $\dot{\gamma}$ reduces to the shear rate and $\dot{\varepsilon}$ vanishes; on uniaxial extensional flow $\dot{\varepsilon}$ reduces to the strain rate and $\dot{\gamma}=\sqrt{3} \dot{\varepsilon}$. Besides, the parameter $\dot{\varepsilon}$ is directly related to the elongational viscosity (Debbaut and Crochet [36]), its definition Eq. (4) is objective and provides a means for its calculation locally in the field (Den Toonder et al. [25]). Further, it is readily related to rheological information and thus measurable, namely through the uniaxial extensional flow. It is an appropriate criterion for turbulent flow and is consistent with the classification and criteria of Hunt et al. [37], with the 
added advantage of possibility to distinguish between stretching and squeezing regions and the ability to quantify their relative strengths. A more detailed analysis of its properties is presented in Den Toonder et al. [25].

Using the following expression from tensorial algebra (Bird et al. [38]:

$$
\operatorname{det} S=\frac{1}{6}\left[(\operatorname{tr} S)^{3}-3(\operatorname{tr} S)\left(\operatorname{tr} S^{2}\right)+2\left(\operatorname{tr} S^{3}\right)\right],
$$

and the incompressibility condition, we can simplify the definition of $\dot{\varepsilon}$ to

$$
\dot{\varepsilon}=\frac{2 \operatorname{tr} S^{3}}{\operatorname{tr} S^{2}}
$$

with $S$ representing here either the instantaneous or the average quantity.

The rate of deformation tensor $\boldsymbol{S}$ is symmetric and its three eigenvalues are real thus imposing restrictions on its invariants (Wilkinson [39]). The eigenvalues of $\boldsymbol{S}$ are the solution of the cubic characteristic equation:

$$
\lambda^{3}+I I_{S} \lambda-I I I_{S}=0,
$$

where the fact that $I_{S}=0$ is already taken into account. The resolving formula for algebraic cubic equations can be found in specialised books on Algebra (e.g. Madureira [40]) and implies that

$$
\left(\frac{I I I_{S}}{2}\right)^{2} \leq\left(-\frac{I I_{S}}{3}\right)^{3}
$$

for the solution to be real. This restriction will be important for the developments in Section 4 .

\subsection{The viscosity function}

As mentioned above, the idea is not to use a complex differential constitutive rheological equation, capable of predicting the measured elongational viscosity, but to include this property into the viscosity function of the simpler generalized Newtonian model through its dependence on the third invariant of the rate of deformation tensor. To this aim, we chose the simplified Carreau-type viscosity model proposed by Davies et al. [41]:

$$
\mu=\mu_{0}\left[1+\left(\lambda_{\mathrm{s}} \dot{\gamma}\right)^{2}\right]^{(n-1) / 2}\left[1+\left(\lambda_{\mathrm{e}} \dot{\varepsilon}\right)^{2}\right]^{(m-1) / 2},
$$

where $\mu_{0}$ is the zero shear-rate viscosity and $\lambda_{\mathrm{s}}$ and $\lambda_{\mathrm{e}}$ are two appropriate time constants.

One of the important conclusions of Den Toonder's et al. [25] work was that the viscosity model needs to be able to predict an increase of the viscosity with both a normal stretching as well as with a normal compression of the molecules, since both contribute to the drag reduction phenomena, whereas earlier theories (Lumley [5]) pointed exclusively to the role of molecular extension. This conclusion is in agreement with the form of the viscosity model (Eq. (9)) because it is an even function on the extension parameter $\dot{\varepsilon}$.

Since the role of shear-thinning was already analysed by Pinho and Whitelaw [42] and confirmed by Collins' predictions [22], who showed the effect to be small and associated with low Reynolds number flows, our attention can concentrate on the effects of the elongational parameter exclusively, so two simplified versions of Eq. (9) are considered: 


$$
\text { Model (1) } \begin{array}{ll}
\mu=\mu_{0} & (\text { for } \dot{\varepsilon}=0), \\
\mu=\mu_{0} K_{\mathrm{e}} & (\text { for } \dot{\varepsilon} \neq 0),
\end{array}
$$

where $\dot{\varepsilon}$ designates instantaneous, average or fluctuating elongational parameters.

This model applies to the solution of the rigid rod-shaped molecules of Fig. 1 (curve 1), which show a constant elongational viscosity regardless of the strain rate value. This is not completely correct, because there should be a transition from a low strain rate Newtonian plateau value of three times the viscometric viscosity. Under turbulent flow conditions, these fluids have strain rates higher than the onset strain rate and this is reflected on the factor $K_{\mathrm{e}}$ in the second of Eq. (10a).

$$
\text { Model (2) } \quad \mu=\mu_{0}\left[1+\left(\lambda_{\mathrm{e}} \dot{\varepsilon}\right)^{2}\right]^{(m-1) / 2} \equiv \mu_{0} g(\dot{\varepsilon}) .
$$

It applies to the behaviour of very flexible molecules at very dilute concentrations, like those of curve 3 in Fig. 1, with an onset of strain-thickening at $1 / \lambda_{\mathrm{e}}$.

Eqs. (10a) and (10b) will only be used whenever essential to proceed with the analysis. The analysis will be kept as general as possible by using the more general definition

$$
\mu=\mu_{0} f(\dot{\gamma}) g(\dot{\varepsilon})
$$

for as long as possible. In Eq. (11), $f()$ and $g()$ are functions yielding the dependence on parameters $\dot{\gamma}$ and $\dot{\varepsilon}$, respectively.

\section{Formulation of the problem}

To show that the inclusion of the third invariant of the rate of deformation tensor into the viscosity model can change the turbulent characteristics of the flows, an order of magnitude analysis of the momentum and of the turbulent kinetic energy transport equations is carried out. First, those equations are adapted to Generalised Newtonian Fluids and the most important new terms to be analysed are identified.

We start by presenting the relevant governing equations, which are based on those in Tennekes and Lumley [43]: the average momentum equation

$$
\rho U_{j} \frac{\partial U_{i}}{\partial x_{j}}=-\frac{\partial p}{\partial x_{i}}+\frac{\partial\left(2 \bar{\mu} S_{i j}+2 \overline{\mu^{\prime} s_{i j}}+2 \overline{\mu^{\prime}} S_{i j}-\rho \overline{u_{i} u_{j}}\right)}{\partial x_{j}},
$$

and the transport equation for the turbulence kinetic energy $\left(k \equiv \frac{1}{2} \overline{u_{i}^{2}}\right)$

$$
\begin{aligned}
\rho U_{j} \frac{\partial k}{\partial x_{j}}= & -\overline{u_{i}} \frac{\partial p}{\partial x_{i}}-\frac{\partial}{\partial x_{j}}\left[\frac{1}{2} \rho \overline{u_{i} u_{i} u_{j}}-2 \bar{\mu} \overline{u_{i} s_{i j}}-2 \overline{\mu^{\prime} u_{i}} S_{i j}-2 \overline{\mu^{\prime} u_{i} s_{i j}}\right]-2 \bar{\mu} \overline{s_{i j}^{2}}-2 \overline{\mu^{\prime} s_{i j}^{2}}-2 \overline{\mu^{\prime} s_{i j}} S_{i j} \\
& -\rho \overline{u_{i} u_{j}} S_{i j} .
\end{aligned}
$$

Here and throughout the paper, capital letters or an overbar represent time-average values of kinematic quantities and of pressure, whereas small letters denote fluctuations (for instance, $U_{i}$ denotes the time-average value of the instantaneous $i$-velocity component and $u_{i}$ its fluctuating term). The time-average of the fluctuating terms of primary kinematic quantities (velocities and rates of deformation) will be zero by definition (Schlichting [44]). A hat $(\wedge)$ will denote 
instantaneous values $\left(\hat{S}_{i j}\right.$ represents the instantaneous value of the $i j$-component of the rate of deformation tensor) and the nomenclature for the viscosity is explained next.

The viscosity is typically a non-linear function of kinematic quantities (Eq. (10b)), and there are at least three approaches for splitting the total viscosity into the sum of an average term and a fluctuating term:

Method 1: $\hat{\mu}=\bar{\mu}(\overline{\bar{\varepsilon}})+\mu^{\prime} ; \quad \overline{\mu^{\prime}} \neq 0$,

where the argument of the average viscosity depends on an average parameter $\overline{\dot{\varepsilon}}$ calculated from the components of the time-average rate of strain tensor. (Note that this definition implies $\overline{\hat{\mu}} \neq \bar{\mu}$ and $\hat{\dot{\varepsilon}} \neq \overline{\bar{\varepsilon}})$

Method 2: $\quad \hat{\mu}=\bar{\mu}\left(\overline{\dot{\varepsilon}}, \dot{\varepsilon}^{\prime}, \dot{\varepsilon}^{\prime 2}, \overline{\dot{\varepsilon}}^{2}, \dot{\varepsilon}^{\prime} \overline{\dot{\varepsilon}}, \dot{\varepsilon}^{\prime 2} \overline{\dot{\varepsilon}}, \dot{\varepsilon}^{\prime} \overline{\dot{\varepsilon}}^{2}, \ldots\right)+\mu^{\prime} ; \quad \overline{\mu^{\prime}}=0$,

where the average viscosity is defined as the time-average, so $\overline{\hat{\mu}}=\bar{\mu}$.

Method 3: $\hat{\mu}=\bar{\mu}(\overline{\hat{\varepsilon}})+\mu^{\prime} ; \quad \overline{\mu^{\prime}} \neq 0$,

where the argument of the viscosity function is the time-average $(\overline{\hat{\varepsilon}})$ of the instantaneous elongation parameter $\hat{\dot{\varepsilon}}$.

The average rate of strain $\overline{\dot{\varepsilon}}$ and the quantity $\dot{\varepsilon}^{\prime}$ appearing in Eqs. (14a), (14b) and (14c) are defined as

$$
\begin{gathered}
\overline{\dot{\varepsilon}} \equiv \frac{2\left(S_{i k} S_{k j}\right) S_{i j}}{S_{i j} S_{i j}}, \\
\dot{\varepsilon}^{\prime} \equiv \frac{2\left(s_{i k} s_{k j}\right) s_{i j}}{s_{i j} S_{i j}} .
\end{gathered}
$$

Method 2 is the traditional approach and implies that the time-average viscosity will be some complex function of the average rate of strain $(\overline{\dot{\varepsilon}})$, of the quantity $\dot{\varepsilon}^{\prime}$, as well as higher order correlations of those two quantities, as indicated in Eq. (14b).

In the order of magnitude analysis it was found more convenient to follow the first method, because it leads to terms involving the usual average viscosity which can then be compared with the corresponding Newtonian terms.

The analysis will concentrate on the magnitude of the following new terms:

(1) from the momentum Eq. (12):

$$
\overline{2 \mu^{\prime} s_{i j}} \text { and } 2 \overline{\mu^{\prime}} S_{i j} \text {. }
$$

(2) and from the turbulence kinetic energy Eq. (13):

$$
2 \overline{\mu^{\prime} u_{i}} S_{i j} ; \quad \overline{2 \mu^{\prime} u_{i} s_{i j}} ;-2 \overline{\mu^{\prime} s_{i j}^{2}} \text { and }-2 \overline{\mu^{\prime} s_{i j}} S_{i j} \text {. }
$$

To proceed further, it is necessary to calculate the various viscosity functions: the instantaneous $(\hat{\mu})$, the average $(\bar{\mu})$ and the fluctuating $\left(\mu^{\prime}\right)$ viscosities. The instantaneous viscosity is given by Eq. (11) with the functions $f()$ and $g()$ expressing in some way (e.g. Eq. (9)) the dependence on the instantaneous values of $\hat{\dot{\gamma}}$ and $\hat{\dot{\varepsilon}}$. Here $\hat{\dot{\varepsilon}}$ is given by Eq. (6) written in terms of $\hat{\boldsymbol{S}}$. 
With the choice of method 1 for averaging (Eq. (14a)), the average viscosity is similarly calculated with $g()$ and $f()$ evaluated with the average values $\overline{\dot{\varepsilon}}$ (Eq. (15a)) and $\overline{\dot{\gamma}}$. Finally, the fluctuating viscosity is the difference between the total (instantaneous) and the average viscosities

$$
\mu^{\prime}=\hat{\mu}-\bar{\mu}=\mu_{0}[f(\hat{\dot{\gamma}}) g(\hat{\dot{\varepsilon}})-f(\overline{\dot{\gamma}}) g(\overline{\dot{\varepsilon}})] .
$$

\section{Order of magnitude analysis in two-dimensional turbulent flows}

The analysis is carried out for the two types of fluids defined by Eqs. (10a) and (10b) and, without loss of generality, two-dimensional flows on the mean will be considered. For two-dimensional flows det $\boldsymbol{S}=0$ and both Eqs. (4) and (15a) yield $\overline{\dot{\varepsilon}}=0$.

In this case, the general viscosity model (Eq. (9)) predicts $g(\overline{\dot{\varepsilon}})=g(0)=1$, therefore

$$
\bar{\mu}=\mu_{0} f(\overline{\dot{\gamma}}) g(0)=\mu_{0} f(\overline{\dot{\gamma}})
$$

and

$$
\mu^{\prime}=\mu_{0} f(\hat{\dot{\gamma}}) g(\hat{\dot{\varepsilon}})-\mu_{0} f(\overline{\dot{\gamma}}) .
$$

\subsection{Fluids with a constant-strain viscosity}

For fluids exhibiting a constant elongational viscosity, and no dependence of the viscosity on $\dot{\gamma}$ (Eq. (10a)), its average and fluctuating values are given by

$$
\begin{aligned}
& \bar{\mu}=\mu_{0}, \\
& \mu^{\prime}=\mu_{0} K_{\mathrm{e}}-\mu_{0} .
\end{aligned}
$$

Starting with the momentum Eq. (12) we conclude that the diffusive term $2 \overline{\mu^{\prime} s_{i j}}$ is zero and the summation of the other two remaining diffusive terms $2 \bar{\mu} S_{i j}+2 \overline{\mu^{\prime}} S_{i j}$ reduces to $2 \mu_{0} K_{\mathrm{e}} S_{i j}$. In conclusion, there is, on the whole, $K_{\mathrm{e}}$ more viscous diffusion than with a fluid showing no non-Newtonian behaviour as far as the elongational viscosity is concerned.

Moving now to the $k$ equation it is obvious that the diffusive term $\left(2 \overline{\mu^{\prime} u_{i}} S_{i j}\right)$ and the dissipative term $\left(2 \overline{\mu^{\prime} s_{i j}} S_{i j}\right)$ listed in Eq. (17) are zero. The remaining diffusive terms of the $k$ equation $\left(2 \overline{\mu^{\prime} u_{i} s_{i j}}\right.$ and $\left.2 \bar{\mu} \overline{u_{i} s_{i j}}\right)$ add to become

$$
2 \overline{\mu^{\prime} u_{i} s_{i j}}+2 \overline{\mu u_{i} s_{i j}}=2 \mu_{0} K_{\mathrm{e}} \overline{u_{i} s_{i j}} \text {. }
$$

This leads to a similar conclusion to that found for the momentum equation, i.e., that the viscous diffusion terms of the $k$ equation are $K_{\mathrm{e}}$ times larger than in the absence of an elongational parameter dependent viscosity. As far as the dissipation of TKE is concerned its two terms add to become

$$
-2 \bar{\mu} \overline{s_{i j}^{2}}-2 \overline{\mu^{\prime} s_{i j}^{2}}=-2 \mu_{0} K_{\mathrm{e}} \overline{s_{i j}^{2}}
$$

a result which is again $K_{\mathrm{e}}$ times larger than that for a strain rate independent viscosity. 


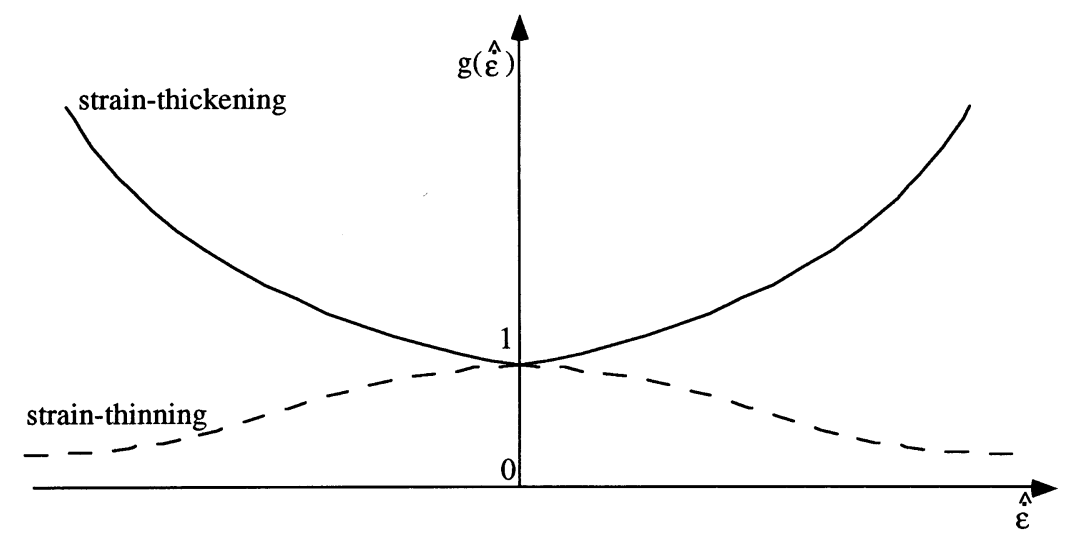

Fig. 2. Variation of a general even viscosity function $g(\hat{\dot{\varepsilon}})$ as a function of the instantaneous elongation parameter $\hat{\dot{\varepsilon}}$.

The viscous diffusion of $k$ is usually quite small in comparison with other terms of its transport equation, except very close to the walls, so a significant effect arising from increased diffusion is not expected. However, that is not the case for the dissipation of $k$; away from the laminar sublayer of a boundary layer, the flow is usually close to equilibrium (production of $k$ equals its dissipation) so an increase in the dissipation of $k$ by a factor of $K_{\mathrm{e}}$ is bound to have a non-negligible indirect effect upon the Reynolds stresses, which are part of the momentum balance. The measurements of $\mathrm{Ng}$ et al. [33] pertaining to a fluid with these characteristics (xanthan gum solution in a thick Newtonian fluid) show a value $K_{\mathrm{e}} \approx 2$. If we further assume that these results are representative of the behaviour of the same additive in a thinner solvent, the analysis indicates the doubling in the levels of the dissipation of $k$. This is certainly bound to have an important effect upon the values of $k$ and in the momentum balance in equilibrium and elsewhere in the flow.

\subsection{Fluids with a strain-thickening viscosity}

We turn now to the viscosity model (Eq. (10b)) which is an even function of the strain rate (see Fig. 2). For two-dimensional flows $\overline{\tilde{\varepsilon}}$ is zero and the average viscosity becomes $\mu_{0}$ (Eq. (19a) with $f(\dot{\gamma})=1$ ). In general, and since $m>1$, regardless of the sign of the instantaneous elongational parameter (positive-stretching or negative-compression), the instantaneous viscosity is always higher than the average viscosity, defining a positive viscosity fluctuation, i.e. from Eq. $(19 b)$,

$$
g(\hat{\hat{\varepsilon}})>1 \Rightarrow \mu^{\prime}>0 \Rightarrow \overline{\mu^{\prime}}>0 .
$$

This fact will help in dealing with the correlations between viscosity fluctuations and fluctuations of other kinematic quantities. Since $\mu^{\prime}$ is an even function of $\hat{\varepsilon}$ it can be expanded in a Taylor series like $\mu^{\prime} / \mu_{0} \sim \lambda_{\mathrm{e}}^{2} \hat{\hat{\varepsilon}}^{2}+$ higher-order even powers of $\hat{\hat{\varepsilon}}$, regardless of the particular form of function $g()$. As a consequence, any correlation of $\mu^{\prime}$ with other approximately Gaussian-distributed variable (like $u_{i}$ or even more so $s_{i j}$, which is related to the small scale of turbulence, Batchelor [45]) will vanish because the odd moments of the normal distribution are 
zero. As a physical interpretation of this we see that, at a given point, positive and negative contributions of the kinematic variable (say, the velocity fluctuations) are equally probable but lead to an always positive similar viscosity fluctuation; when the contributions are added, the net result is a vanishing correlation. Statistically speaking, the bivariate pdf of $\mu^{\prime}$ and of the kinematic quantity (say, $s_{i j}$ ) is symmetric about $s_{i j}=0$ which implies $\mu^{\prime} s_{i j}=0$ although the two variables are not independent (Libby [46]). Thus

$$
\overline{\mu^{\prime} s_{i j}} \approx 0 \text {. }
$$

Finally, looking at the momentum equation, we see that the additional diffusive term $2 \overline{\mu^{\prime}} S_{i j}$ adds to the standard term $2 \bar{\mu} S_{i j}$ since $\overline{\mu^{\prime}}$ is always positive, and the modelled momentum equation for two-dimensional flows becomes

$$
\rho U_{j} \frac{\partial U_{i}}{\partial x_{j}}=-\frac{\partial p}{\partial x_{i}}+\frac{\partial\left[2\left(\bar{\mu}+\overline{\mu^{\prime}}\right) S_{i j}-\rho \overline{u_{i} u_{j}}\right]}{\partial x_{j}} .
$$

The new terms in the $k$ Eq. (13) are investigated next. The first diffusive term is simplified on the same grounds as in Eq. (23) to become

$$
2 \overline{\mu^{\prime} u_{i}} S_{i j} \approx 0
$$

where the arguments exposed above now apply to $\mu^{\prime}$ and $u_{i}$.

The fluctuating viscosity $\mu^{\prime}$ is always positive, so the second diffusive term $2 \overline{\mu^{\prime} u_{i} s_{i j}}$ can be simplified, in terms of order of magnitude, into

$$
2 \overline{\mu^{\prime} u_{i} s_{i j}} \approx 2 \overline{\mu^{\prime}} \overline{u_{i} s_{i j}}
$$

This decoupling is related to the simplification of the next term. The sign of the term represented in Eq. (26) is the same as that of the classical diffusive term $2 \bar{\mu} \overline{u_{i} s_{i j}}$ based on the average viscosity. These two terms pertain to molecular diffusion, which anyway are less important than other turbulent contributions.

There are also two additional contributions of dissipative nature in the $k$ equation: the term

$$
2 \overline{\mu^{\prime} s_{i j}} S_{i j} \approx 0
$$

is solved as implied in Eq. (23), whereas the remaining term $\left(2 \overline{\mu^{\prime} s_{i j}^{2}}\right)$ is always positive, because both $\mu^{\prime}$ and $s_{i j}^{2}$ are real positive even functions. In consequence it acts in the same direction as the standard turbulence dissipation rate $\left(2 \bar{\mu} \overline{s_{i j}^{2}}\right)$. This term $\left(2 \overline{\mu^{\prime} s_{i j}^{2}}\right)$ can be approximated as

$$
2 \overline{\mu^{\prime} s_{i j}^{2}} \approx 2 \overline{\mu^{\prime}} \overline{s_{i j}^{2}}
$$

based on the following arguments. If $\mu^{\prime}$ and $s_{i j}^{2}$ are uncorrelated the equality sign applies in Eq. (28); however, this is not expected to be the case since $\mu^{\prime}$ is a function of $\hat{\dot{\varepsilon}}^{2}$, which is itself proportional to $s_{i j}^{2}$, and the average on the left hand side of Eq. (28) turns out to be related to the fourth moment of the strain rate. According to Batchelor [45], the strain rate is a variable with an approximately Gaussian distribution, therefore the second term in Eq. (28) will be multiplied by 3 (where 3 is the kurtosis of the Gaussian distribution). In any case, by assuming Eq. (28) as it is, the correct term is being underpredicted by a factor of around 3, therefore the analysis is being carried out on the safe side. 
In conclusion, the following approximate $k$ equation for two-dimensional turbulent flow is obtained

$$
\rho U_{j} \frac{\partial k}{\partial x_{j}}=-\overline{u_{i} \frac{\partial p}{\partial x_{i}}}-\frac{\partial}{\partial x_{j}}\left[\frac{1}{2} \rho \overline{u_{i} u_{i} u_{j}}-2\left(\bar{\mu}-\overline{\mu^{\prime}}\right) \overline{u_{i} s_{i j}}\right]-2\left(\bar{\mu}+\overline{\mu^{\prime}}\right) \overline{s_{i j}^{2}}-\rho \overline{u_{i} u_{j}} S_{i j},
$$

with $\overline{\mu^{\prime}}$ taking only positive values.

The remaining issue is the determination of the value of $\overline{\mu^{\prime}}$ relative to $\bar{\mu}$ and this requires an order of magnitude analysis of the argument of the function $g()$ to be performed in the next section. Nevertheless, it is at least already clear from Section 4.1 that, regardless of the value of $\overline{\mu^{\prime}}$, provision for a third invariant dependence of the viscosity, within the GNF model, can strongly affect the turbulence equations, especially the dissipation of turbulence.

\subsubsection{The magnitude of $\hat{\dot{\varepsilon}}$ in two-dimensional turbulent flows}

In order to quantify the ratio $\overline{\mu^{\prime}} / \bar{\mu}$, the particular function $g(\hat{\dot{\varepsilon}})$ (Eq. (10b))

$$
g(\hat{\dot{\varepsilon}})=\left[1+\left(\lambda_{\mathrm{e}} \hat{\dot{\varepsilon}}\right)^{2}\right]^{(m-1) / 2}
$$

has to be analysed in more detail. This function is even on the elongation parameter and to assess its order of magnitude it is necessary to estimate that of the instantaneous elongation rate as well as typical values for the parameters $\lambda_{\mathrm{e}}$ and $m$. For strain-thickening fluids $(m>1)$ the function $g($ ) increases from 1 , the value at zero $\hat{\dot{\varepsilon}}$, and decreases from 1 for strain-thinning fluids $(m<1)$ (see Fig. 2). Parameter $m$ measures the rate at which function $g()$ changes with the elongational parameter, and $\lambda_{\mathrm{e}}$ is the inverse of the strain rate at which the function $g()$ changes from the Newtonian low strain rate plateau onto the power law region. Function $g($ ) plays the role of the constant $K_{\mathrm{e}}$ for the constant elongational viscosity model (Eq. (10a)), namely, that of representing the ratio of the Trouton-ratio of the fluid to the Trouton-ratio of the solvent.

Possible values of the parameter $m$ can be estimated from the work of $\mathrm{Ng}$ et al. [33]. For the very flexible $50 \mathrm{ppm}$ polyacrylamide solution, $m$ increases from about 1.15 , for extensional rates between 1 and $10 \mathrm{~s}^{-1}$, to about 1.43 at extensional rates between 10 and $100 \mathrm{~s}^{-1}$, and it increases further for higher strain rates. So, two typical values of $m$ are 1.5 and 1.1, representing two different cases, and are chosen here. For the time constant $\left(\lambda_{\mathrm{e}}\right)$ the selection is slightly more difficult. The same measurements with the polyacrylamide solutions indicate a value close to 1 $\mathrm{s}$, of the order of $0.1 \mathrm{~s}$ for the CMC solution and above $1 \mathrm{~s}$ (say, $10 \mathrm{~s}$ ) for the xanthan gum solution. So, these three possibilities for $\lambda_{\mathrm{e}}$ will be tried.

The argument of $g()$ in Eq. (30) is complex because it contains many non-linear terms as readily apparent if $\hat{\dot{\varepsilon}}$ is replaced with expression (Eq. (6)) written in terms of $\hat{\boldsymbol{S}}$. If the numerator of expression (Eq. (6)) for $\hat{\dot{\varepsilon}}$ is expanded, various terms arise yielding both positive and negative contributions and it is not easy to ascertain what will be the order of magnitude of its total. However, if the elongation parameter is expressed on the basis of the eigenvalues of the rate of deformation tensor (Den Toonder et al. [25]) it can be concluded that $\dot{\varepsilon}$ will be positive when the element of fluid is being compressed in two directions and stretched in one, the so-called uniaxial extension, and negative when the element of fluid is being compressed. The vortex stretching in turbulent flows is made of many uniaxial extensions and constitutes one of the main kinematic events of turbulence (Bradshaw [47]). The DNS simulations of Den Toonder et al. [26] have also shown that squeezing of elements of fluid can be more important for the non-Newtonian features of turbulent flow than stretching. 
Anyway, it should be stressed that we are looking at the time-average value of the function $g()$, which will be related with the values of $\hat{\dot{\varepsilon}}$, because $g()$ is an even function of this parameter. Thus, it is important to estimate the maximum possible value of $\hat{\dot{\varepsilon}}$ and to this aim two alternative approaches can be taken. One is based on the direct evaluation of the magnitude of $\hat{\dot{\varepsilon}}$ in the argument of $g()$ and is given in Appendix A, where it is shown to lead to a similar result as that of the preferred approach here explained.

This second approach follows from inequality (Eq. (8)), an algebraic result reflecting that solutions of the characteristic equation of the rate of deformation tensor are real. This result also holds for the instantaneous tensor $\hat{\boldsymbol{S}}$;

$$
\left(\frac{I I I_{\hat{S}}}{2}\right)^{2} \leq\left(-\frac{I I_{\hat{S}}}{3}\right)^{3},
$$

from which we can obtain a maximum value of the elongation parameter $\hat{\dot{\varepsilon}}$. Replacing the definition of the invariants in Eq. (31) we get

$$
\operatorname{tr} \hat{\boldsymbol{S}}^{3} \leq \frac{\left(\operatorname{tr} \hat{\boldsymbol{S}}^{2}\right)^{3 / 2}}{\sqrt{6}}
$$

Since definition (Eq. (6)) is also valid for $\hat{\dot{\varepsilon}}$ in terms of the instantaneous tensor $\hat{\boldsymbol{S}}$ we have, upon substitution of Eq. (32) into Eq. (6), the maximum value of the elongation rate parameter

$$
\hat{\dot{\varepsilon}} \leq \sqrt{\frac{2}{3} \operatorname{tr} \hat{\boldsymbol{S}}^{2}}=\frac{\hat{\dot{\gamma}}}{\sqrt{3}} \Rightarrow \hat{\dot{\varepsilon}}^{2} \leq \frac{2}{3} \operatorname{tr} \hat{\boldsymbol{S}}^{2} .
$$

It is worth noting that this inequality becomes an equality for the kinematic situation of a pure elongational flow, a likely instantaneous situation for a portion of fluid in turbulent flow.

The trace of the squared instantaneous rate of deformation tensor in Eq. (33) can be expanded as

$$
\operatorname{tr} \hat{\boldsymbol{S}}^{2}=\hat{S}_{i j} \hat{S}_{i j}=\left(S_{i j}+s_{i j}\right)\left(S_{i j}+s_{i j}\right)=S_{i j} S_{i j}+2 S_{i j} S_{i j}+S_{i j} S_{i j}=\operatorname{tr} \hat{\boldsymbol{S}}^{2}+2 S_{i j} S_{i j}+\operatorname{tr} \boldsymbol{s}^{2} .
$$

In order to simplify this expression we invoke a standard argument of high Reynolds number turbulent flows (cf. Tennekes and Lumley [43] and Batchelor [45]), namely that there is very little direct interaction between the strain rate fluctuations, which are related to the small scales of turbulence, and the mean flow. This is synonymous to saying that the magnitudes of the fluctuations of the rate of deformation are much higher than the corresponding values of the average rate of deformation, i.e.

$$
\sqrt{s_{i j}^{2}} \gg\left|S_{i j}\right| \text {. }
$$

In this equation, the summation convention to repeated indices does not apply; it is the root mean square value of $s_{i j}$ that is being considered. In this case, all terms of first and second order in $S_{i j} / s_{i j}$ in Eq. (34) can be neglected and the outcome is

$$
\operatorname{tr} \hat{S}^{2} \approx \operatorname{tr} s^{2} \text {. }
$$

Note that in the definition of $\operatorname{tr} \boldsymbol{s}^{2}$ all terms are positive, and so there is no question of cancellation when assuming that both $S_{i j} S_{i j}$ and $2 S_{i j} s_{i j}$ are much smaller than $s_{i j} s_{i j}$. Considering that in turbulent flows all components of the fluctuating rate of deformation tensor are of 
similar magnitude $\left(\sqrt{s_{i j}^{2}} \approx s\right.$, again no summation over $i$ and $j$ ), and working in the principal axis required for an order of magnitude analysis which is independent of the system of reference then, in the limit

$$
\boldsymbol{O}\left(\overline{\operatorname{tr} \hat{S}^{2}}\right) \approx \boldsymbol{O}\left(\overline{s_{i j} s_{i j}}\right) \approx 3 s^{2} .
$$

Eq. (33) can be averaged and majored, to become

$$
\boldsymbol{O}\left(\overline{\hat{\dot{\varepsilon}}^{2}}\right) \leq 2 s^{2} \text {. }
$$

From this, the maximum value of the average function $g()$ is related to the maximum of its argument by

$$
\overline{g(\hat{\dot{\varepsilon}})}=\overline{\left[1+\left(\lambda_{\mathrm{e}} \hat{\dot{\varepsilon}}\right)^{2}\right]^{(m-1) / 2}} \leq\left[1+\lambda_{\mathrm{e}}^{2} \overline{\hat{\dot{\varepsilon}}}^{2}\right]^{(m-1) / 2},
$$

with

$$
1+\lambda_{\mathrm{e}}^{2 \overline{\hat{\varepsilon}^{2}}} \leq 1+\lambda_{\mathrm{e}}^{2} \alpha s^{2}
$$

where the constant $\alpha$ takes the value $\alpha=2$ from Eq. (38).

\subsubsection{The fluctuating viscosity $\overline{\left(\mu^{\prime}\right)}$ in two-dimensional turbulent flows}

With the maximum possible value of $\alpha$ being 2 from Eqs. (39a) and (39b), a single value of $\alpha=1$ will be considered next for the estimation of $\overline{\mu^{\prime}} / \bar{\mu}$. This parameter is combined with three different values of $\lambda_{\mathrm{e}}$, namely $\lambda_{\mathrm{e}}=0.01 \mathrm{~s}, \lambda_{\mathrm{e}}=1 \mathrm{~s}$ and $\lambda_{\mathrm{e}}=10 \mathrm{~s}$, together with two values for the exponent $m, m=1.1$ and $m=1.5$. Finally, it will be necessary to use adequate estimates of $s$, which are derived on the basis of Newtonian turbulent pipe flow for cross-sectional average conditions and near-wall conditions.

Following Durst et al. [48], the area-average rate of energy dissipation in a pipe $\langle\varepsilon\rangle$ is simply given by the work of the wall shear force per unit mass:

$$
\langle\varepsilon\rangle=\frac{\tau_{w} \pi D L\langle U\rangle}{\rho \frac{\pi}{4} L D^{2}},
$$

where $\langle U\rangle$ and $D$ are the bulk velocity and the pipe diameter, respectively. If this is equated with the definition of the mean rate of energy dissipation,

$$
\varepsilon \equiv 2 v \overline{s_{i j} s_{i j}}=2 v \operatorname{tr} s^{2}=6 v s^{2},
$$

the following estimate for an area-average $s$ is obtained:

$$
\frac{s D}{\langle U\rangle}=\sqrt{\frac{f \mathrm{Re}}{12}},
$$

where $f$ is the Darcy friction coefficient. For water flows at Reynolds numbers between 30000 and $10^{5}$, in 25 and $50 \mathrm{~mm}$ diameter pipes, the average $s$ is in the range $200 \mathrm{~s}^{-1}$ to $2000 \mathrm{~s}^{-1}$ and the values of the area-average $s$ increase with the fluid viscosity, at constant Reynolds numbers.

Maximum values of $s$ occur near the wall and can be estimated as follows. In thin boundary layers, production of turbulent kinetic energy $(k)$ is mainly balanced by its dissipation $(\varepsilon)$ so, following Libby [46] and a $k-\varepsilon$ formulation 


$$
\varepsilon=\sqrt{c_{\mu}} k \frac{\partial\langle U\rangle}{\partial y},
$$

where the universal constant $c_{\mu}=0.09$. Near the wall, the velocity profile follows the linear viscous sublayer law and the limiting values of the rms of the velocity fluctuations are, according to Durst et al. [48] and Kim et al. [49],

$$
\sqrt{u^{2}}=0.37\langle U\rangle ; \quad \sqrt{w^{2}}=0.21\langle U\rangle ; \quad \sqrt{v^{2}}=0,
$$

thus giving $k=0.09\langle U\rangle^{2}=c_{\mu}\langle U\rangle^{2}$. After combining these equations with the definition of $\varepsilon$ above, the following normalised relation for the value of $s$ at the wall is obtained

$$
\frac{s D}{\langle U\rangle}=\operatorname{Re} c_{\mu}^{3 / 4} \sqrt{\frac{f}{48}} .
$$

For the same water flows as above the value of $s$ at the pipe wall is in the range $1300 \mathrm{~s}^{-1}$ to $50000 \mathrm{~s}^{-1}$, with its maximum value increasing with the fluid viscosity, at constant Reynolds numbers. Other estimates of $s$ at the wall can be evaluated alternatively, as suggested in Libby [46], but they yield similar values and therefore are not included.

In view of these estimates, it seems appropriate to select a value of $s=1000 \mathrm{~s}^{-1}$ for the remaining analysis, but to take into account rather low values of the Reynolds number, which are prone to occur for very viscous fluids, a value of $s=100 \mathrm{~s}^{-1}$ will also be considered.

4.2.2.1. Case $\lambda_{e}=0.01 s^{-1}$. For such a low value of $\lambda_{\mathrm{e}}$, then the term $\lambda_{\mathrm{e}}^{2} s^{2}$ is larger than 1 unless $s$ is very low. Substituting, for $m=1.5$ into

$$
\begin{aligned}
\overline{g(\hat{\dot{\varepsilon}})}=\overline{\left[1+\lambda_{\mathrm{e}}^{2} \hat{\dot{\varepsilon}}^{2}\right]^{(m-1) / 2}} & \approx\left[1+0.01^{2} \alpha s^{2}\right]^{(1.5-1) / 2}, \\
\overline{g(\hat{\dot{\varepsilon}})}=\left[1+0.01^{2} \alpha s^{2}\right]^{0.25} & \Rightarrow \text { for } s=100 \mathrm{~s}^{-1}, \overline{g(\hat{\hat{\varepsilon}})} \approx 1.19, \\
& \Rightarrow \text { for } s=1000 \mathrm{~s}^{-1}, \quad \overline{g(\hat{\hat{\varepsilon}})} \approx 3.17 .
\end{aligned}
$$

For the lower $m=1.1, \overline{g(\hat{\hat{\varepsilon}})} \approx 1.035$ and 1.26 corresponding to the case of Eq. (46a) and (46b), respectively. Fluids with a very late onset of strain-thickening are also likely to exhibit low values of the exponent $m$, so a result of around $\overline{g(\hat{\varepsilon})} \approx 1.15$ obtained with $m=1.1$ should be closer to reality.

4.2.2.2. Case $\lambda_{e}=1 s^{-1}$. Now, also with $m=1.5$,

$$
\overline{g(\hat{\dot{\varepsilon}})}=\overline{\left[1+\lambda_{\mathrm{e}}^{2} \hat{\dot{\varepsilon}}^{2}\right]^{(m-1) / 2}} \approx\left[1+\alpha s^{2}\right]^{(1.5-1) / 2} .
$$

In this case, $\alpha s^{2} \gg 1$, since $\alpha=1$, and

$$
\begin{aligned}
\overline{g(\hat{\dot{\varepsilon}})} \approx \sqrt{s} & \Rightarrow \text { for } s=100 \mathrm{~s}^{-1}, \quad \overline{g(\hat{\dot{\varepsilon}})} \approx 10, \\
& \Rightarrow \text { for } s=1000 \mathrm{~s}^{-1}, \quad \overline{g(\hat{\dot{\varepsilon}})} \approx 31.6 .
\end{aligned}
$$

With the lower value of $m=1.1$ the outcome would be $\overline{g(\hat{\hat{\varepsilon}})} \approx 1.58$ and 2.0 , respectively. Obviously, the earlier onset of strain-thickening leads to a more intense effect upon the fluctuating viscosity not just because of the higher $\lambda_{\mathrm{e}}$, but also because the value of $m$ is bound to be higher than 1.1 and smaller than 1.5. Even if an upper bound in the elongation of the 
molecules is accounted for, $g(\hat{\dot{\varepsilon}})$ can easily rise to about 4 or 5 , certainly a very important effect of similar magnitude to that encountered with the constant elongational viscosity fluids.

4.2.2.3. Case $\lambda_{e}=10 s^{-1}$. This corresponds to an earlier onset of strain-thickening in practice leading to the highest values of $m$ and consequently of the fluctuating viscosities. For $m=1.5$, function $g()$ becomes

$$
\overline{g(\hat{\hat{\varepsilon}})}=\overline{\left[1+\lambda_{\mathrm{e}}^{2} \hat{\dot{\varepsilon}}^{2}\right]^{(m-1) / 2}} \approx\left[1+100 \alpha s^{2}\right]^{(1.5-1) / 2} .
$$

With $100 \alpha s^{2} \gg 1$ and $\alpha=1$, the result is

$$
\begin{aligned}
\overline{g(\hat{\dot{\varepsilon}})} \approx \sqrt{10 s} & \Rightarrow \text { for } s=100 \mathrm{~s}^{-1}, \quad \overline{g(\hat{\dot{\varepsilon}})} \approx 31.6, \\
& \Rightarrow \text { for } s=1000 \mathrm{~s}^{-1}, \quad \overline{g(\hat{\dot{\varepsilon}})} \approx 100,
\end{aligned}
$$

These are indeed very large values of the viscosity function. For a smaller value of the exponent $(m=1.1)$ the resulting values of $\overline{g(\hat{\dot{\varepsilon}})}$ would be 2.0 and 2.5 , respectively. Thus, even for this case the strain-thickening effect is considerable, leading to an average viscosity double of the corresponding strain-rate independent viscosity.

\subsection{Three-dimensional turbulent flows}

The characterisation of function $g()$ is more difficult in this case, because the average value of the elongation parameter will not be zero. There are many flows of practical relevance which are norminally three-dimensional but have a strong two-dimensional character in most of the flow field, as far as average quantities are concerned, such as the case of flows with boundarylayers. The conclusions of the previous sections are clear: provision for a viscosity dependent on the third invariant of the rate of deformation tensor is important and has effects on those terms of the transport equations containing the viscosity fluctuations, that range from a few percent to hundreds percent relative to the role of similar terms involving the average viscosity. If that increase is irrelevant for the viscous diffusive terms at high Reynolds number flows, it is bound to have a strong effect in the dissipation of turbulence kinetic energy and of the individual Reynolds stresses and thus will affect indirectly the momentum equations. In three-dimensional flows this type of analysis is more complicated and will not be carried out here, but it is expected to yield similar conclusions.

\section{Discussion}

For constant elongational viscosity fluids, it was demonstrated that the new terms in the equations induce increased diffusion and dissipation of turbulence kinetic energy by a factor of $K_{\mathrm{e}}$, where $K_{\mathrm{e}}$ is the ratio of the Trouton number of the solution to that of the pure solvent. For $K_{\mathrm{e}}$ of about 2, as in some of the measurements of $\mathrm{Ng}$ et al. [33], then the dissipation increases by a factor of 2 relative to that of fluids in which viscosity is not a function of $I I I_{S}$.

For variable elongational viscosity fluids, the parameter $K_{\mathrm{e}}$ is substituted by function $g($ ), with the results now depending on the magnitude of the instantaneous elongation parameter $(\hat{\dot{\varepsilon}})$. 
The maximum value of the elongation parameter is obtained from the mathematical requirement of real eigenvalues for the instantaneous rate of deformation tensor. It is shown that even for values of the elongation parameter considerably lower than the maximum, there is an increase of dissipative effects due to the dependence of $\mu$ on the third invariant of $\hat{\boldsymbol{S}}$. Only for very low magnitudes of fluctuations of the instantaneous elongation parameter, unlikely to be encountered in real turbulent flows, will the elongational viscosity of fluids of variable viscosity be lower than that pertaining to the constant elongational viscosity fluids. For high fluctuating strain rates, the increase of viscosity of flexible polymers is stronger than that encountered for rigid molecules of constant elongational viscosity, and the present analysis predicts a considerable effect upon the turbulence dissipation. The measurements of Tirtaamadja and Sridhar [32], where transient Trouton ratios of the order of 1000 are reported for very flexible molecules, are considerably higher than the apparent elongational viscosity measured with the opposed jet rheometer by $\mathrm{Ng}$ et al. [33] and used here to estimate $n$ and $\lambda_{\mathrm{e}}$. Thus, the present results of the effect of the elongational viscosity on turbulence may actually be conservative.

In this analysis, strain-thickening fluids were considered, but the arguments and some conclusions also apply to many tension-thinning fluids, as well. Continuum mechanics (Barnes et al. [50]) shows that, at the limit of zero strain rates, non-Newtonian elastic liquids behave as Newtonian fluids that follow the relation

$$
\frac{\partial \eta_{\mathrm{e}}}{\partial \dot{\varepsilon}}>0
$$

Thus, tension-thickening will always exist, but at higher strain rates the fluid can be tension-thinning. Quite often real fluids are shear-thinning, and also exhibit a tension-thinning behaviour at high strain rates, but what matters is that their Trouton ratio is in fact monotonically increasing with either the shear rate or the strain rate. In this work, we have emphasised that the increased dissipative and diffusive effects on turbulence are actually to be related to an increased Trouton ratio rather than to strain-thickening, although here the former was shown through the latter (Fig. 1). The basis for comparison was always the viscosity at the average strain rate, but similar conclusions would be obtained for elastic fluids having a shearand tension-thinning behaviour, provided their Trouton ratio is larger than 3 .

There are various ways to introduce a third invariant dependence in the constitutive equation. It is possible to follow the same approach as in here (Eq. (10b)), but with a different viscosity function, such as one of those used by Debbaut and Crochet [36], which exhibit a more realistic variation of the elongational viscosity with the elongation parameter in that the slope of the curve increases with the strain rate. Alternatively, it is possible to adopt the idea of Orlandi [27], where an anisotropic effect acts on the material function of the normal stresses. More recently, an anisotropic viscosity perspective was also investigated by Den Toonder et al. [26] in their DNS calculations, who confirmed the advantages relative to an isotropic model. The elongation parameter could also be differently defined; instead of Eq. (4) a different parameter could be used but it should at least obey objectivity and frame invariance. Whatever the choices, all these approaches are bound to affect the turbulence equations, and especially their dissipation term, in different magnitudes but in the same direction as found here.

By using the transport equation of the turbulence kinetic energy in the order of magnitude investigation, we selected a representative quantity of turbulence and somehow hinted at a $k-\varepsilon$ 
type of turbulence model, but similar conclusions would be reached with the transport equations for the Reynolds stresses. These equations (Libby [46]) possess terms representing the dissipation of each specific Reynolds stress, which have a very similar mathematical description as the dissipation of $k$ and so conclusions would be identical.

This paper also hints at possible ways of modelling turbulence with similar viscosity model equations together with the $k-\varepsilon$ or the Reynolds stress models. The transport equations of the turbulence quantities will have new extra terms involving the fluctuations of the viscosity, which need to be properly handled for closure. The main issue here is the appearance of parameters $(\dot{\gamma})$ and $(\dot{\varepsilon})$, which must be related to other quantities in the equations, or separately modelled. The relationship between $\dot{\gamma}$ and $\dot{\varepsilon}$ and with the fluctuations of the rate of deformation tensor and the rate of dissipation of turbulence kinetic energy found here suggests a possible strategy for closure of two-equation or higher-order turbulence models, which will be the subject of a future report.

\section{Conclusions}

The literature survey in Section 1 has shown that the traditional approach for turbulence modelling of drag reducing non-Newtonian fluid flows, based on purely viscous fluids and modified wall functions, has not had great success and that an approach based on more realistic constitutive equations becomes necessary.

With the choice of a generalised Newtonian viscosity model, and with provision for this viscosity to depend on the third invariant of the rate-of-deformation tensor, an order of magnitude analysis of the new terms of the momentum and turbulence kinetic energy transport equations was undertaken. The parameters of the rheological model were estimated from results of recent measurements of the elongational viscosity and the outcome of this qualitative investigation was that the dissipation of turbulent kinetic energy was increased by a factor that could easily be of the order of $50 \%$, going up to hundreds of percent, relative to that of a third invariant independent viscosity.

The observed trend is in the right direction, as is also demonstrated by the DNS results of Orlandi [27] and Den Toonder et al. [25,26], and encourages further work aimed at developing single-point coupled rheological-turbulence models.

The relationship between the shear parameter, the elongation parameter, the fluctuations of the rate of deformation tensor and the rate of dissipation of turbulence kinetic energy indicate a possible strategy for implementing a two-equation or a higher-order turbulence model for the prediction of non-Newtonian drag-reducing fluids.

\section{List of symbols}

$f($ ) Component of the viscosity function accounting for a shear rate dependence

$g($ ) Component of the viscosity function accounting for a strain rate dependence

$I_{S}, I I_{S}$ and First, second and third invariants of the rate of deformation tensor

$I I I_{S}$

$k \quad$ Turbulent kinetic energy 


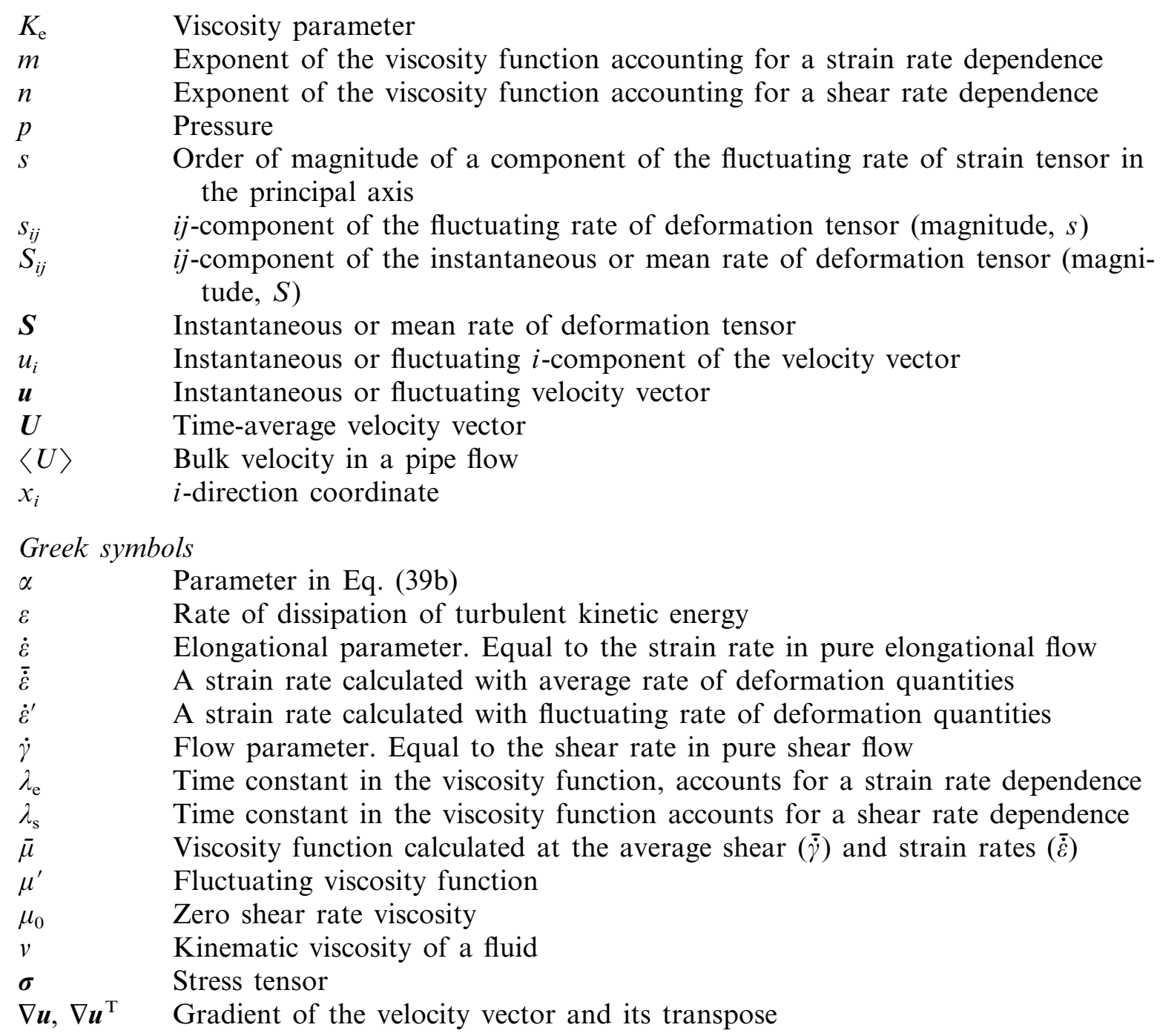

\section{Acknowledgements}

The authors would like to acknowledge the helpful comments of the referees. The authors are listed alphabetically.

\section{Appendix A. Order of magnitude of function $g()$ - an alternative approach}

An alternative approach to estimate the order of magnitude of function $g($ ) stems directly from an analysis of its argument, without making use of the restriction imposed by the characteristic equation of the rate of deformation tensor. The viscosity fluctuation is, from Eq. 
(18) with $f(\hat{\dot{\gamma}})=f(\overline{\dot{\gamma}})=1$,

$$
\mu^{\prime}=\mu_{0} g\left[\frac{2 \operatorname{tr} \hat{\boldsymbol{S}}^{3}}{\operatorname{tr} \hat{\boldsymbol{S}}^{2}}\right]-\mu_{0},
$$

and, upon expanding the numerator and taking into account that $\operatorname{tr} \boldsymbol{S}^{3}=0$ for two-dimensional flow, we obtain

$$
\operatorname{tr} \hat{\boldsymbol{S}}^{3}=\left(S_{i k} S_{k j}\right) s_{i j}+\left[S_{i k} s_{k j}+s_{i k} S_{k j}\right]\left(S_{i j}+s_{i j}\right)+\left(s_{i k} S_{k j}\right) S_{i j}+\operatorname{tr} \boldsymbol{s}^{3} .
$$

Under turbulent flow conditions, the order of magnitude analysis for $\operatorname{tr} \hat{\boldsymbol{S}}^{3}$, follows the same steps as that of $\operatorname{tr} \hat{\boldsymbol{S}}^{2}$ in the main text. Second order terms on $S_{i j}$ smaller than $\operatorname{tr} \boldsymbol{s}^{3}$ are

$$
\left(S_{i k} S_{k j}\right) s_{i j} \ll \operatorname{tr} s^{3}, \quad\left(S_{i k} s_{k j}\right) S_{i j} \ll \operatorname{tr} s^{3} \quad \text { and } \quad\left(s_{i k} S_{k j}\right) S_{i j} \ll \operatorname{tr} s^{3},
$$

and first order terms smaller than $\operatorname{tr} s^{3}$ are

$$
\left(s_{i k} s_{k j}\right) S_{i j}<\operatorname{tr} s^{3}, \quad\left(S_{i k} s_{k j}\right) s_{i j}<\operatorname{tr} s^{3} \quad \text { and } \quad\left(s_{i k} S_{k j}\right) s_{i j}<\operatorname{tr} s^{3} .
$$

A bit of algebra demonstrates that, due to the symmetry of tensors $\boldsymbol{s}$ and $\boldsymbol{S}$

$$
\left(S_{i k} s_{k j}\right) s_{i j}=\left(s_{i k} S_{k j}\right) s_{i j}=\left(s_{i k} s_{k j}\right) S_{i j}
$$

and

$$
\left(S_{i k} S_{k j}\right) s_{i j}=\left(s_{i k} S_{k j}\right) S_{i j}=\left(S_{i k} S_{k j}\right) S_{i j}
$$

The various terms in Eqs. (A3a) and (A3b) have positive and negative contributions, but since we need to take the square of Eq. (A1), we retain all terms to yield

$$
\operatorname{tr} \hat{\boldsymbol{S}}^{3}=3\left(S_{i k} S_{k j}\right) s_{i j}+3\left(s_{i k} s_{k j}\right) S_{i j}+\operatorname{tr} \boldsymbol{s}^{3},
$$

where Eqs. (A3a) and (A3b) was used. For $\operatorname{tr} \hat{\boldsymbol{S}}^{2}$ we shall use Eq. (34) of the main text.

Since in general the function $g()$ is even on the parameter $\hat{\dot{\varepsilon}}$, we can use the approximation $\overline{g(\hat{\dot{\varepsilon}})} \leq g\left(\hat{\dot{\varepsilon}}^{2}\right)$ and majorate $\overline{\hat{\varepsilon}}^{2}$. The different contributions to $\operatorname{tr} \hat{\boldsymbol{S}}^{3}$ in $\hat{\dot{\varepsilon}}$ can be positive or negative, but still we can overestimate its maximum value if we assume that all $s_{i j}$ and $S_{i j}$ are of similar magnitude and positive. To this aim, we write $s=\sqrt{s_{i j}^{2}}$ (no summation) and $S=\sqrt{S_{i j}^{2}}$ (no summation), and the order of magnitude analysis in the principal axis $\left(\operatorname{tr} s^{2}=3 s^{2}\right.$ and $\operatorname{tr} s^{3}=$ $\left.3 s^{3}\right)$ gives

$$
\overline{\operatorname{tr} \widehat{S}^{3}} \approx 3\left(3 s^{2} S+s^{3}+3 S^{2} s\right)
$$

and

$$
\operatorname{tr} \hat{S}^{2} \approx 3\left(S^{2}+s^{2}+2 S s\right)
$$

so that the magnitude of the argument of the $g$ ( )-function becomes 


$$
1+\lambda_{\mathrm{e}}^{2} \overline{\hat{\dot{\varepsilon}}}^{2} \approx 1+\lambda_{\mathrm{e}}^{2} 4 s^{2}\left[\frac{1+6 \frac{S}{s}+15\left(\frac{S}{s}\right)^{2}+18\left(\frac{S}{s}\right)^{3}+9\left(\frac{S}{s}\right)^{4}}{1+4 \frac{S}{S}+6\left(\frac{S}{s}\right)^{2}+4\left(\frac{S}{s}\right)^{3}+\left(\frac{S}{s}\right)^{4}}\right] .
$$

Further progress would require a relation between fluctuating and average rates of deformation enabling Eq. (A6) to be recasted as

$$
1+\alpha \lambda^{2} s^{2}
$$

For $s \approx 10 S$ one would end up with $\alpha$ equal to 4.83 , whereas with a more realistic estimate of $s \approx 100 S \alpha$ equals 4.08. If we compare the results of the two alternative approaches to estimate the argument of $g()$ and $\overline{\mu^{\prime}}$, respectively, Eqs. (39a), (39b) and (A7), we see that they are in reasonable agreement if in Eq. (A7) we take $s \approx 100 S$ as required by Eq. (35). The agreement between two approaches based on completely different starting arguments lends support to the correctness of the estimate for maximum $(\hat{\dot{\varepsilon}})$. The arguments leading to Eqs. (39a) and (39b) are more correct and account for both positive and negative contributions in the brackets of Eq. (A1), so expression Eqs. (39a) and (39b) is preferred for the estimates of Section 4.2.2.

\section{References}

[1] N.S. Berman, H. Tan, Two-component laser-Doppler velocimeter studies of submerged jets of dilute polymer solutions, AIChE J. 2 (1985) 208.

[2] O.S. Castro, F.T. Pinho, Tubulent expansion flow of low molecular weight shear-thinning solutions, Exp. Fluids $20(1995) 42$.

[3] J.W. Hoyt, The effect of additives on fluid friction, J. Basic Eng. 94 (1972) 258.

[4] P.S. Virk, Drag reduction fundamentals, AIChE J. 21 (1975) 625.

[5] J.L. Lumley, Drag reduction in two-phase and polymer flows, Phys. Fluids 20 (1977) S64.

[6] M.M. Reischman, W.G. Tiederman, Laser-Doppler anemometer measurements in drag-reducing channel flows, J. Fluid Mech. 70 (1975) 369.

[7] T.S. Luchik, W.G. Tiederman, Turbulent structure in low concentration drag-reducing channel flows, J. Fluid Mech. 190 (1988) 241.

[8] H.-W. Bewersdorff, N.S. Berman, The influence of flow-induced non-Newtonian fluid properties on turbulent drag reduction, Rheol. Acta 27 (1988) 130.

[9] R.G. Larson, Constitutive Equations for Polymer Melts and Solutions, Butterworths, Boston, 1988.

[10] I. Radin, J.L. Zakin, G.K. Patterson, Drag reduction in solid-fluid systems, AIChE J. 21 (1975) 358.

[11] H. Chanson, Drag reduction in open channel flow by aeration and suspended load, J. Hydraulic Res. 32 (1994) 87.

[12] H.-W. Bewersdorff, Heterogene Widerstandsverminderung bei turbulenten Rohrströmungen, Rheol. Acta 23 (1984) 522.

[13] G. Ryskin, Turbulent drag reduction by polymers: a quantitative theory, Phys. Rev. Lett. 59 (1987) 2059.

[14] P.G. De Gennes, Towards a scaling theory of drag reduction, Physica 140A (1986) 9.

[15] M. Tabor, C.J. Durning, B. O’Shaughnessy, The microscopic origins of drag reduction. Internal Report of the Department of Applied Physics, Columbia University, New York, 1989.

[16] Y. Kawaguchi, Y. Tawaraya, A. Yabe, K. Hishida, M. Maeda, Active control of turbulent drag reduction in surfactant solutions by wall heating, Proceedings of the Symposium on Turbulence Modification and Drag Reduction, ASME, San Diego, 1966. 
[17] A. Steiff, K. Klöpper, Application of Drag Reducing Additives in District Heating Systems, ASME Fluids Engineering Division Conference, vol. 237, 1966, p. 235.

[18] F. Durst, A.K. Rastogi, Calculations of turbulent boundary-layer flows with drag reducing polymer additives, Phys. Fluids 20 (1977) 1975.

[19] S. Hassid, M. Poreh, A turbulent energy dissipation model for flows with drag reduction, ASME J. Fluids Eng. 100 (1978) 107.

[20] F. Durst, T. Keck, R. Kleine, Turbulence quantities and Reynolds stress in pipe flow of polymer solutions measured, Proceedings of the Sixth Symposium on Turbulence in Liquids, University of Missouri-Rolla, 1979, p. 55 .

[21] S. Politis, Turbulence modelling of inelastic power-law fluids, Technical Report. No. 52, Brite project RIIB.0085.UK (H), Imperial College of Science and Technology, 1989.

[22] P.J. Collins, Prediction of turbulent flow of inelastic shear-thinning fluids in ducts and mixing vessels, $\mathrm{PhD}$. Thesis, Imperial College, University of London, UK, 1990.

[23] G. Astarita, F. Marrucci, Non-Newtonian Fluid Mechanics, McGraw-Hill, 1974.

[24] C.J.S. Petrie, Extensional flow: a mathematical perspective, Rheol. Acta 34 (1995) 12.

[25] J.M.J. Den Toonder, F.T.M. Nieuwstadt, G.D.C. Kuiken, The role of elongational viscosity in the mechanism of drag reduction by polymer additives, Appl. Sci. Res. 54 (1995) 95.

[26] J.M.J. Den Toonder, M.A. Hulson, G.D.C. Kuiken, F.T.M. Nieuwstadt, Drag reduction by polymer additives in a turbulent pipe flow: numerical and laboratory experiments, J. Fluid Mech. 337 (1997) 193.

[27] P. Orlandi, A tentative approach to the direct simulation of drag reduction by polymers, J. Non-Newtonian Fluid Mech. 60 (1995) 277.

[28] F. Durst, R. Haas, W. Interthal, Laminar and turbulent flows of dilute polymer solutions: a physical model, Rheol. Acta 21 (1982) 572.

[29] N.E. Hudson, T.E.R. Jones, The A1 project-An overview, J. Non-Newtonian Fluid Mech. 46 (1993) 69.

[30] P. Dontula, M. Pasquali, L.E. Scriven, C.W. Macosko, Can extensional viscosity be measured with opposed nozzle devices?, Rheol. Acta 36 (1997) 429.

[31] C.G. Hermansky, D.V. Boger, Opposing-jet viscometry of fluids with viscosity approaching that of water, J. Non-Newtonian Fluid Mech. 56 (1995) 1.

[32] V. Tirtaatmadja, T. Sridhar, A filament stretching device for measurement of extensional viscosity, J. Rheol. 37 (1993) 1081.

[33] S.L. Ng, R.P. Mun, D.V. Boger, D.F. James, Extensional viscosity measurements of dilute solutions of various polymers, J. Non-Newtonian Fluid Mech. 65 (1996) 291.

[34] N.S. Berman, Drag reduction of the highest molecular weight fractions of polyethylene oxide, Phys. Fluids 20 (1977) 715.

[35] P.S. Virk, H.S. Mickley, K.A. Smith, The ultimate asymptote and mean flow structure in Tom's phenomena, J. Appl. Mech. 92 (1970) 488.

[36] B. Debbaut, M.J. Crochet, Extensional effects in complex flows, J. Non-Newtonian Fluid Mech. 30 (1988) 169.

[37] J.C.R. Hunt, A.A. Wray, P. Moin, Eddies, streams and convergence zones in turbulent flows, Center for Turbulence Research, Proceedings of the Summer Program, 1988, p. 193.

[38] R.B. Bird, R. Armstrong, O. Hassager, Dynamics of polymeric liquids, vol. 2, Fluid Dynamics, 2nd ed., John Wiley, New York, 1987.

[39] J.H. Wilkinson, The Algebraic Eigenvalue Problem, Clarendon Press, Oxford, 1965.

[40] A. Madureira, Lessons in Superior Algebra and Analytical Geometry, vol. 1, Algebra, 2nd ed., Porto Editora, 1948 (in Portuguese).

[41] A.R. Davies, I.M. Farah, M. Rides, K. Thomas, Numerical evaluation of a method for determining extensional viscosity of fluids using contraction flow analysis, Makromol. Chem. Macromol. Symp. 68 (1993) 25.

[42] F.T. Pinho, J.H. Whitelaw, Flow of non-Newtonian fluids over a confined baffle, J. Fluid Mech. 226 (1991) 475.

[43] H. Tennekes, J.L. Lumley, A first course in turbulence, The MIT Press, Massachussets, USA, 1972.

[44] H. Schlichting, Boundary-Layer Theory, McGraw-Hill, New York, 1969.

[45] G.K. Batchelor, The Theory of Homogeneous Turbulence, Cambride University Press, 1953. 
[46] P.A. Libby, Introduction to Turbulence, Taylor and Francis, New York, 1996, p. 274 and pp. 288-290.

[47] P. Bradshaw, An Introduction to Turbulence and its Measurements, Pergamon Press, Oxford, 1971.

[48] F. Durst, J. Jovanovic, J. Sender, LDA measurements in the near-wall region of a turbulent pipe flow, J. Fluid Mech. 295 (1995) 305.

[49] J. Kim, P. Moin, R. Moser, Turbulence statistics in a fully developed channel flow at low Reynolds number, J. Fluid Mech. 177 (1987) 133.

[50] H. Barnes, J.F. Hutton, K. Walters, An Introduction to Rheology, Elsevier, Oxford, 1989. 